\title{
Ticagrelor schützt wirksamer vor einem erneuten Schlaganfall als ASS
}

Fragestellung: Ist in der frühen Sekundärprävention bei Patienten mit transitorischer ischämischer Attacke (TIA) oder ischämischem Insult arteriosklerotischer Genese Ticagrelor wirksamer als Acetylsalicylsäure (ASS)?

Hintergrund: Die Sekundärprävention des Schlaganfalls bei Patienten ohne kardiale Emboliequelle erfolgt mit Thrombozytenfunktionshemmern, bevorzugt mit ASS. Die SOCRATESStudie hatte bei 13.199 Patienten mit TIA oder leichtem Schlaganfall Ticagrelor mit ASS verglichen. Es zeigte sich eine Risikoreduktion für den kombinierten Endpunkt aus Schlaganfall, Herzinfarkt und Tod zugunsten von Ticagrelor, wobei der Unterschied aber knapp die Signifikanz verpasste [1].

Ein häufiger Fehler bei der Planung von Sekundärpräventionsstudien ist, dass Patienten mit unterschiedlicher Ätiologie des Schlaganfalls eingeschlossen werden. So ist beispielsweise bei Patienten mit lakunären Infarkten, die ganz überwiegend durch schlecht eingestellte Hypertonie und Diabetes mellitus bedingt sind, keine dramatische Wirkung von Thrombozytenfunktionshemmern zu erwarten. Diese Patienten waren allerdings in der SOCRATES-Studie eingeschlossen worden. Idealerweise sollten potentere Thrombozytenfunktionshemmer als

Amarenco P, Albers GW, Denison $\mathrm{H}$ et al. Efficacy and safety of ticagrelor versus aspirin in acute stroke or transient ischaemic attack of atherosclerotic origin: a subgroup analysis of SOCRATES, a randomised, double-blind, controlled trial. Lancet Neurol 2017; 16: 301 - 10
ASS bei Patienten mit nachgewiesener Arteriosklerose eingesetzt werden. Dies wurde in der vorliegenden Subgruppenanalyse der SOCRATES-Studie ausgewertet.

Patienten und Methodik: SOCRATES war eine randomisierte, doppelblinde, kon- trollierte Studie, bei der Patienten mit Hochrisiko-TIA und leichtem Schlaganfall, die älter als 40 Jahre waren und keine kardiale Emboliequelle aufwiesen, mit Ticagrelor oder ASS behandelt wurden. Die Patienten wurden innerhalb von 24 Stunden randomisiert und erhielten eine initiale Dosis von $180 \mathrm{mg} \mathrm{Ti}$ cagrelor am Tag 1 und zweimal 90 mg über die nächsten 90 Tage. Die initiale Dosis von ASS betrug 300 mg und die Langzeitdosis $100 \mathrm{mg}$ täglich. Der primäre Endpunkt waren Schlaganfall, Myokardinfarkt und Tod innerhalb von 90 Tagen. In der Subgruppenanalyse wurden 3.081 Patienten rekrutiert, die eine nachgewiesene Arteriosklerose der hirnversorgenden Arterien aufwiesen. Diese Patienten stellten $23 \%$ der Gesamtpopulation dar.

Ergebnisse: In der Ticagrelor-Gruppe erreichten 103 von 1.542 Patienten, entsprechend 6,7\%, den primären Endpunkt, verglichen mit 147 von 1.539 entsprechend 9,6\% der Patienten in der ASS-Gruppe. Dies entspricht einer Hazard Ratio [HR] von 0,68 , die mit einem $\mathrm{p}$-Wert von 0,03 signifikant war. Bei den $\mathrm{Pa}-$ tienten ohne nachgewiesener ipsilateraler Stenose der hirnversorgenden Arterien betrug die HR 0,97 und war nicht signifikant. In der Untergruppe der Patienten mit nachgewiesener Arteriosklerose ergaben sich keine Unterschiede bei lebensbedrohlichen schwerwiegenden oder geringen Blutungskomplikationen zwischen den beiden Therapien.

Schlussfolgerungen: In einer präspezifizierten Subgruppenanalyse zeigte sich, das Ticagrelor im Vergleich zu ASS bei Patienten mit nachgewiesener Arteriosklerose der hirnversorgenden Arterien signifikant wirksamer ist bezüglich der Verhinderung von Schlaganfällen, Myokardinfarkten und der Sterblichkeit.

\section{- Kommentar von Hans-Christoph Diener, Essen}

\section{Der absolute Nutzen ist nicht sehr hoch}

Leider lassen sich zwei Designfehler der SOCRATES-Studie jetzt nicht mehr rückgängig machen:

- Für die Studie war ein kombinierter Endpunkt aus Schlaganfall, Myokardinfarkt und Tod definiert worden. Allerdings ist aus früheren Studien mit Thrombozytenfunktionshemmern bekannt, dass wegen der geringen Inzidenz in der Regel weder Herzinfarkte noch Todesfälle durch die Behandlung reduziert werden können und lediglich das Gesamtergebnis verwässern. Hätte man allein den Endpunkt Schlaganfall betrachtet, wäre das Ergebnis der SOCRATES-Studie positiv gewesen.

- Es macht nicht sehr viel Sinn, Patienten mit unterschiedlichen Schlaganfallätiologien in Studien einzuschließen, bei denen potentere Thrombozytenfunktionshemmer als Acetylsalicylsäure untersucht werden.

Die vordefinierte Subgruppenanalyse, die hier publiziert wurde, zeigt überzeugend, dass Patienten mit nachgewiesener Arteriosklerose der hirnversorgenden Arterien tatsächlich einen Nutzen von Ticagrelor im Vergleich zu Acetylsalicylsäure haben. Allerdings ist der absolute Nutzen bezüglich ischämischer Schlaganfälle mit 33 verhinderten Ereignissen nicht sehr hoch und die zusätzlichen Kosten wären erheblich.

\footnotetext{
Referenz:

1. Johnston SC et al. N Engl J Med 2016; 375: 35 - 43
} 